JOURNAL OF THE

AMERICAN MATHEMATICAL SOCIETY

Volume 21, Number 3, July 2008, Pages 909-924

S 0894-0347(07)00589-9

Article electronically published on November 29, 2007

\title{
NEW UPPER BOUNDS FOR KISSING NUMBERS FROM SEMIDEFINITE PROGRAMMING
}

\author{
CHRISTINE BACHOC AND FRANK VALLENTIN
}

\section{INTRODUCTION}

In geometry, the kissing number problem asks for the maximum number $\tau_{n}$ of unit spheres that can simultaneously touch the unit sphere in $n$-dimensional Euclidean space without pairwise overlapping. The value of $\tau_{n}$ is only known for $n=1,2,3,4,8,24$. While its determination for $n=1,2$ is trivial, it is not the case for other values of $n$.

The case $n=3$ was the object of a famous discussion between Isaac Newton and David Gregory in 1694. For a historical perspective of this discussion we refer to 6]. The first valid proof of the fact " $\tau_{3}=12$ ", as in the icosahedron configuration, was only given in 1953 by K. Schütte and B.L. van der Waerden in [23].

In the 1970s, P. Delsarte developed a method, initially aimed at bounding codes on finite fields (see [8) that yields an upper bound for $\tau_{n}$ as a solution of a linear program and more generally yields an upper bound for the size of spherical codes of given minimal distance. We shall refer to this method as the LP method. With this method, A.M. Odlyzko and N.J.A. Sloane ([16]), and independently V.I. Levenshtein ([14]), proved $\tau_{8}=240$ and $\tau_{24}=196560$ which are, respectively, the number of shortest vectors in the root lattice $E_{8}$ and in the Leech lattice. For other values of $n$, the LP method gives in many cases the best known upper bounds. However, for $n=3$ and $n=4$ it only gives the upper bounds $\tau_{3} \leq 13$ and $\tau_{4} \leq 25$.

In 2003, O.R. Musin succeeded in proving the conjectured value $\tau_{4}=24$, which is the number of shortest vectors in the root lattice $D_{4}$, with a variation of the LP method (see [15] and the survey [19] of F. Pfender and G.M. Ziegler).

To complete the picture, let us discuss uniqueness of the optimal point configurations. For dimensions 8 and 24, uniqueness was proved by E. Bannai and N.J.A. Sloane (3]). Their proof exploits the fact that the LP method obtains exactly the aimed value. For dimension 3 , there are infinitely many possible configurations. In the regular icosahedron configuration, the angular distances between the contact points are strictly greater than the required $\pi / 3$; hence these points can be moved around obtaining infinitely many new suitable configurations. This

Received by the editors October 17, 2006.

2000 Mathematics Subject Classification. Primary 52C17, 90C22.

Key words and phrases. Spherical codes, kissing number, semidefinite programming, orthogonal polynomials.

The second author was supported by the Netherlands Organization for Scientific Research under grant NWO 639.032.203 and by the Deutsche Forschungsgemeinschaft (DFG) under grant SCHU 1503/4-1. 
partially explains why the determination of $\tau_{3}$ is difficult. On the contrary, uniqueness of the optimal configuration of points in dimension 4 is widely believed, but it remains unproven.

The LP method, which was established by P. Delsarte, J.M. Goethals and J.J. Seidel in [9, handles the more general problem of the determination of a bound for the maximal number

$$
A(n, \theta)=\max \left\{\operatorname{card}(C): C \subset S^{n-1} \text { with } c \cdot c^{\prime} \leq \cos \theta \text { for } c, c^{\prime} \in C, c \neq c^{\prime}\right\}
$$

of points on the unit sphere with minimal angular distance $\theta$. Such configurations of points, also called spherical codes with minimal angular distance $\theta$, are of special interest in information theory. The kissing number problem is equivalent to the problem of finding $A(n, \pi / 3)$.

In this paper, we define a semidefinite program (SDP for short) whose optimal solution gives an upper bound for $A(n, \theta)$ and strengthens the LP method. Computational results show that for several values of $n$ this SDP method gives better upper bounds for $\tau_{n}$ than the LP method.

To be more precise, let us recall that the LP method relies on the existence of polynomials $P_{k}^{n}(t)$, satisfying the so-called positivity property:

$$
\text { for all finite } C \subset S^{n-1}, \sum_{\left(c, c^{\prime}\right) \in C^{2}} P_{k}^{n}\left(c \cdot c^{\prime}\right) \geq 0 .
$$

These polynomials arise as zonal spherical polynomials on the sphere, i.e. the zonal polynomials associated to the decomposition of the space of polynomial functions under the action of the orthogonal group $\mathrm{O}\left(\mathbb{R}^{n}\right)$.

The consideration of the action restricted to a subgroup $H$ of $\mathrm{O}\left(\mathbb{R}^{n}\right)$, chosen to be the stabilizer group of a fixed point $e \in S^{n-1}$, leads us to some symmetric matrices $S_{k}^{n}$ whose coefficients are symmetric polynomials in three variables such that

$$
\text { for all finite } C \subset S^{n-1}, \sum_{\left(c, c^{\prime}, c^{\prime \prime}\right) \in C^{3}} S_{k}^{n}\left(c \cdot c^{\prime}, c \cdot c^{\prime \prime}, c^{\prime} \cdot c^{\prime \prime}\right) \succeq 0
$$

where the sign " $\succeq 0$ " stands for "is positive semidefinite". The reason why we obtain matrices instead of functions comes from the fact that, in the decomposition of the space of polynomial functions on the sphere under the action of $H$, multiplicities greater than 1 appear. In fact these multiplicities are exactly the sizes of the corresponding matrices. From (11) and (2) we derive an SDP whose solution gives an upper bound for $A(n, \theta)$.

Our approach adapts the method proposed by A. Schrijver in 22 to the unit sphere whereas he obtains new upper bounds for binary codes from an SDP. His work can also be interpreted in group theoretic terms, involving the isometry group of the Hamming space $\mathbb{F}_{2}^{n}$ and the subgroup stabilizing $(0, \ldots, 0)$ which is the group of permutations of the $n$ positions. It is very likely that many other spaces of interest in coding theory can be treated likewise. The case of non-binary codes was considered by D.C. Gijswijt, A. Schrijver and H. Tanaka in [11.

The paper is organized as follows: Section 2 reviews on the LP method. Section 3 introduces and calculates the semidefinite zonal matrices associated to the action of $H$ and leading to the matrices $S_{k}^{n}$. Section 4 defines the semidefinite program and its dual that establishes the desired bound. Section 5 discusses computational results. 


\section{REVIEW OF THE LP METHOD ON THE UNIT SPHERE}

We introduce the following notation. The standard inner product of the Euclidean space $\mathbb{R}^{n}$ is denoted by $x \cdot y$. The unit sphere

$$
S^{n-1}:=\left\{x \in \mathbb{R}^{n}: x \cdot x=1\right\}
$$

is homogeneous under the action of the orthogonal group $\mathrm{O}\left(\mathbb{R}^{n}\right)=\left\{O \in \mathbb{R}^{n \times n}\right.$ : $O^{t} O=I_{n}$, where $I_{n}$ denotes the identity matrix. It is moreover two-point homogeneous, meaning that the orbits of $\mathrm{O}\left(\mathbb{R}^{n}\right)$ on pairs of points are characterized by the value of their inner product. The space of real polynomial functions of degree at most $d$ on $S^{n-1}$ is denoted by $\operatorname{Pol}_{\leq d}\left(S^{n-1}\right)$. It is endowed with the induced action of $\mathrm{O}\left(\mathbb{R}^{n}\right)$ and equipped with the standard $\mathrm{O}\left(\mathbb{R}^{n}\right)$-invariant inner product

$$
(f, g)=\frac{1}{\omega_{n}} \int_{S^{n-1}} f(x) g(x) d \omega_{n}(x),
$$

where $\omega_{n}=\frac{2 \pi^{n / 2}}{\Gamma(n / 2)}$ is the surface area of $S^{n-1}$ for the standard measure $d \omega_{n}$. It is a well-known fact (see e.g. [24, Ch. 9.2]) that under the action of $\mathrm{O}\left(\mathbb{R}^{n}\right)$

$$
\mathrm{Pol}_{\leq d}\left(S^{n-1}\right)=H_{0}^{n} \perp H_{1}^{n} \perp \ldots \perp H_{d}^{n},
$$

where $H_{k}^{n}$ is isomorphic to the $\mathrm{O}\left(\mathbb{R}^{n}\right)$-irreducible space

$$
\operatorname{Harm}_{k}^{n}=\left\{f \in \mathbb{R}\left[x_{1}, \ldots, x_{n}\right]: f \text { homogeneous, deg } f=k, \sum_{i=1}^{n} \frac{\partial^{2}}{\partial x_{i}^{2}} f=0\right\}
$$

of harmonic polynomials in $n$ variables which are homogeneous and have degree $k$. We set $h_{k}^{n}:=\operatorname{dim}\left(\operatorname{Harm}_{k}^{n}\right)=\left(\begin{array}{c}n+k-1 \\ n-1\end{array}\right)-\left(\begin{array}{c}n+k-3 \\ n-1\end{array}\right)$.

A certain family of orthogonal polynomials is associated to the unit sphere. They will be denoted by $P_{k}^{n}$, with the convention that $P_{k}^{n}$ has degree $k$ and is normalized by $P_{k}^{n}(1)=1$. For $n \geq 3$ these polynomials are up to multiplicative constants Gegenbauer polynomials $C_{k}^{\lambda}$ with parameter $\lambda=n / 2-1$. So they are given by $P_{k}^{n}(t)=C_{k}^{n / 2-1}(t) / C_{k}^{n / 2-1}(1)$, and the Gegenbauer polynomials $C_{k}^{\lambda}$ can be inductively defined by $C_{0}^{\lambda}(t)=1, C_{1}^{\lambda}(t)=2 \lambda t$, and

$$
k C_{k}^{\lambda}(t)=2(k+\lambda-1) t C_{k-1}^{\lambda}(t)-(k+2 \lambda-2) C_{k-2}^{\lambda}(t), \quad \text { for } k \geq 2 .
$$

They are orthogonal with respect to the weight function $\left(1-t^{2}\right)^{\lambda-1 / 2}$ on the interval $[-1,1]$. For $n=2$ the polynomials $P_{k}^{n}$ coincide with the Chebyshev polynomials of the first kind $T_{k}$ which can be inductively defined by $T_{0}(t)=1, T_{1}(t)=t$, and

$$
T_{k}(t)=2 t T_{k-1}(t)-T_{k-2}(t), \quad \text { for } k \geq 2,
$$

and they are orthogonal with respect to the weight function $\left(1-t^{2}\right)^{-1 / 2}$ on the interval $[-1,1]$.

The polynomials $P_{k}^{n}(t)$ are related to the decomposition (3) by the so-called addition formula (see e.g. [1, Ch. 9.6]): for any orthonormal basis $\left(e_{1}, \ldots, e_{h_{k}^{n}}\right)$ of $H_{k}^{n}$ and for any pair of points $x, y \in S^{n-1}$ we have

$$
P_{k}^{n}(x \cdot y)=\frac{1}{h_{k}^{n}} \sum_{i=1}^{h_{k}^{n}} e_{i}(x) e_{i}(y)
$$


From the addition formula (4), the positivity property (1) becomes obvious:

$$
\begin{aligned}
\sum_{\left(c, c^{\prime}\right) \in C^{2}} P_{k}^{n}\left(c \cdot c^{\prime}\right) & =\sum_{\left(c, c^{\prime}\right) \in C^{2}} \frac{1}{h_{k}^{n}} \sum_{i=1}^{h_{k}^{n}} e_{i}(c) e_{i}\left(c^{\prime}\right) \\
& =\frac{1}{h_{k}^{n}} \sum_{i=1}^{h_{k}^{n}} \sum_{\left(c, c^{\prime}\right) \in C^{2}} e_{i}(c) e_{i}\left(c^{\prime}\right)=\frac{1}{h_{k}^{n}} \sum_{i=1}^{h_{k}^{n}}\left(\sum_{c \in C} e_{i}(c)\right)^{2} \geq 0 .
\end{aligned}
$$

Now we introduce the unknowns of the LP to be considered. For a spherical code $C$ we define the two-point distance distribution

$$
x(u):=\frac{1}{\operatorname{card}(C)} \operatorname{card}\left\{\left(c, c^{\prime}\right) \in C^{2}: c \cdot c^{\prime}=u\right\},
$$

where $u \in[-1,1]$. Clearly, only a finite number of $x(u)$ 's are not equal to zero, and the positivity property can be rewritten as a linear inequality in the $x(u)^{\prime}$ 's:

$$
\sum_{u \in[-1,1]} x(u) P_{k}^{n}(u) \geq 0 .
$$

Moreover, the number of elements of $C$ is given by $\operatorname{card}(C)=\sum_{u \in[-1,1]} x(u)$. Noticing the obvious conditions $x(1)=1, x(u) \geq 0$, and $x(u)=0$ for $\cos \theta<u<1$ if the minimal angular distance of $C$ is $\theta$, we are led to consider the following linear program: For any $d \geq 1$, the optimal solution of the linear program

$$
\begin{aligned}
& \max \left\{1+\sum_{\begin{array}{l}
u \in[-1, \cos \theta] \\
\end{array}} x(u)=0 \text { for all but finitely many } u \in[-1, \cos \theta],\right. \\
& x(u) \geq 0 \text { for all } u \in[-1, \cos \theta], \\
&\left.1+\sum_{u \in[-1, \cos \theta]} x(u) P_{k}^{n}(u) \geq 0 \text { for all } k=1, \ldots, d\right\}
\end{aligned}
$$

gives an upper bound for $A(n, \theta)$. The dual linear problem is

$$
\begin{aligned}
\min \left\{1+\sum_{k=1}^{d} f_{k} \quad:\right. \\
f_{k} \geq 0 \text { for all } k=1, \ldots, d \\
\left.\quad \sum_{k=1}^{d} f_{k} P_{k}^{n}(u) \leq-1 \text { for all } u \in[-1, \cos \theta]\right\} .
\end{aligned}
$$

By the duality theorem (cf. [10]) any feasible solution of (7) gives an upper bound for the optimal solution of (6). The dual linear program can be restated in the following way involving polynomials:

Theorem 2.1 (See e.g. [9, Th. 4.3], [12, [16, 7, Ch. 9]). Let $F(t)=\sum_{k=0}^{d} f_{k} P_{k}^{n}(t)$ be a polynomial of degree at most $d$ in $\mathbb{R}[t]$. If

(a) $f_{k} \geq 0$ for all $k \geq 1$ and $f_{0}>0$ and then

(b) $F(u) \leq 0$ for all $u \in[-1, \cos \theta]$,

$$
A(n, \theta) \leq \frac{F(1)}{f_{0}}
$$




\section{SEMidefinite ZONAL MATRICES}

Now we fix a point $e \in S^{n-1}$ and let $H:=\operatorname{Stab}\left(\mathrm{O}\left(\mathbb{R}^{n}\right), e\right)$ be the stabilizer of $e$ in $\mathrm{O}\left(\mathbb{R}^{n}\right)$. Obviously, $H \simeq \mathrm{O}\left(\mathbb{R}^{n-1}\right)$ since $\mathrm{O}\left(\mathbb{R}^{n-1}\right)$ can be identified with the orthogonal group of the orthogonal complement of $\mathbb{R} e$.

It is a classical result (see e.g. [24, Ch. 9.2]) that for the restricted action to $H$ the decomposition of $\operatorname{Harm}_{k}^{n}$ into $H$-irreducible subspaces is given by

$$
\operatorname{Harm}_{k}^{n} \simeq \bigoplus_{i=0}^{k} \operatorname{Harm}_{i}^{n-1}
$$

Hence, each of the $H_{k}^{n}$ in (3) decomposes likewise:

$$
H_{k}^{n}=H_{0, k}^{n-1} \perp H_{1, k}^{n-1} \perp \ldots \perp H_{k, k}^{n-1},
$$

where $H_{i, k}^{n-1} \simeq \operatorname{Harm}_{i}^{n-1}$. We give an explicit description of this decomposition in the proof of Theorem 3.2

We summarize the situation in the following picture:

$$
\begin{array}{rllllllll}
\mathrm{Pol}_{\leq d}\left(S^{n-1}\right)= & H_{0}^{n} & \perp & H_{1}^{n} & \perp & \ldots & \perp & H_{d}^{n} \\
= & H_{0,0}^{n-1} & \perp & H_{0,1}^{n-1} & \perp & \ldots & \perp & H_{0, d}^{n-1} \\
& & & \perp & H_{1,1}^{n-1} & \perp & \ldots & \perp & H_{1, d}^{n-1} \\
& & & & & \ldots & \ldots & \ldots & \ldots
\end{array} .
$$

The isotypic components of the $H$-decomposition of $\mathrm{Pol}_{\leq d}\left(S^{n-1}\right)$ are

$$
\mathcal{I}_{k}:=H_{k, k}^{n-1} \perp \ldots \perp H_{k, d}^{n-1} \simeq(d-k+1) \operatorname{Harm}_{k}^{n-1}, \quad \text { for } k=0, \ldots, d .
$$

Now we show how to associate to each $\mathcal{I}_{k}$ a "zonal matrix" in view of an analogue of the addition formula (4).

Theorem 3.1. Let $\mathcal{I}=R_{0} \perp R_{1} \perp \ldots \perp R_{m} \simeq(m+1) R$ be an isotypic component of $\mathrm{Pol}_{\leq d}\left(S^{n-1}\right)$ under the action of $H$, with $R$ an $H$-irreducible space of dimension $h$. Let $\left(e_{0,1}, \ldots, e_{0, h}\right)$ be an orthonormal basis of $R_{0}$ and let $\phi_{i}: R_{0} \rightarrow R_{i}$ be $H$ isomorphisms preserving the inner product on $\operatorname{Pol}_{\leq d}\left(S^{n-1}\right)$. Let $e_{i, j}=\phi_{i}\left(e_{0, j}\right)$, so that $\left(e_{i, 1}, \ldots, e_{i, h}\right)$ is an orthonormal basis of $R_{i}$. Define

$$
E(x):=\left(\frac{1}{\sqrt{h}} e_{i, j}(x)\right)_{\substack{0 \leq i \leq m \\
1 \leq j \leq h}}=\frac{1}{\sqrt{h}}\left(\begin{array}{ccc}
e_{0,1}(x) & \ldots & e_{0, h}(x) \\
\vdots & & \vdots \\
e_{m, 1}(x) & \ldots & e_{m, h}(x)
\end{array}\right)
$$

and

$$
Z(x, y):=E(x) E(y)^{t} \in \mathbb{R}^{(m+1) \times(m+1)} .
$$

Then the following properties hold for the matrix $Z$ :

(a) $Z(x, y)$ does not depend on the choice of the orthonormal basis of $R_{0}$.

(b) The change of $\phi_{i}$ to $-\phi_{i}$ for some $i$ or the choice of another decomposition of $\mathcal{I}$ as a sum of $m+1$ orthogonal $H$-submodules changes $Z(x, y)$ to some $O Z(x, y) O^{t}$ with $O \in \mathrm{O}\left(\mathbb{R}^{m+1}\right)$.

(c) For all $g \in H, Z(g(x), g(y))=Z(x, y)$.

(d) (Matrix-type positivity property)

$$
\text { For all finite } C \subset S^{n-1}, \sum_{\left(c, c^{\prime}\right) \in C^{2}} Z\left(c, c^{\prime}\right) \succeq 0 .
$$


Proof. (a) If $\left(\epsilon_{0,1}, \ldots, \epsilon_{0, h}\right)$ is another orthonormal basis of $R_{0}$, then there is an orthogonal $h \times h$ matrix $O$ with $\left(\epsilon_{0,1}, \ldots, \epsilon_{0, h}\right)=\left(e_{0,1}, \ldots, e_{0, h}\right) O$. In this case the matrix $E(x)$ is changed to $E(x) O$ and, since $O O^{t}=I_{h}$, the matrix $Z(x, y)$ stays unchanged.

(b) By Schur's Lemma and by the irreducibility of $R$, there are only two possible choices for $\phi_{i}$, namely $\phi_{i}$ and $-\phi_{i}$, once the subspaces $R_{i}$ are fixed.

Let $\mathcal{I}=S_{0} \perp \ldots \perp S_{m}$ be another decomposition of $\mathcal{I}$, together with $H$ isomorphisms $\psi_{i}: R_{i} \rightarrow S_{i}$ preserving the inner product on $\mathrm{Pol}_{\leq d}\left(S^{n-1}\right)$. Then $\psi=\left(\psi_{0}, \ldots, \psi_{m}\right)$ defines an $H$-endomorphism of $\mathcal{I}$. Again by Schur's Lemma, for a suitable choice of basis in $R_{i}$ and by permuting rows and columns, the matrix of $\psi$ is block diagonal with $h$ blocks of size $(m+1) \times(m+1)$ and with the same $(m+1) \times(m+1)$ matrix $O \in \mathrm{O}\left(\mathbb{R}^{m+1}\right)$ as blocks. This means that $E(x)$ changes to $O E(x)$ and so $Z(x, y)$ becomes $O Z(x, y) O^{t}$.

(c) Since

$$
e_{i, j}\left(g^{-1}(x)\right)=\left(g e_{i, j}\right)(x),
$$

the computation of $Z\left(g^{-1}(x), g^{-1}(y)\right)$ amounts to replacing the $e_{i, j}$ by $g e_{i, j}$ in the definition of $Z(x, y)$. Since $R_{i}$ is $H$-stable, $\epsilon_{i, j}:=g e_{i, j}$, with $j=1, \ldots, h$, is another orthonormal basis of $R_{i}$, and

$$
\phi_{i}\left(\epsilon_{1, j}\right)=\phi_{i}\left(g e_{1, j}\right)=g \phi_{i}\left(e_{1, j}\right)=g e_{i, j}=\epsilon_{i, j} .
$$

Hence from (a) we conclude $Z\left(g^{-1}(x), g^{-1}(y)\right)=Z(x, y)$.

(d) We have $\sum_{\left(c, c^{\prime}\right) \in C^{2}} Z\left(c, c^{\prime}\right)=\left(\sum_{c \in C} E(c)\right)\left(\sum_{c \in C} E(c)\right)^{t} \succeq 0$.

The orbits of $H$ on pairs of points on the unit sphere $x, y \in S^{n-1}$ are characterized by the values of the three inner products $e \cdot x, e \cdot y$, and $x \cdot y$. By definition the coefficients $Z_{i, j}(x, y)$ of $Z(x, y)$ are polynomials in the variables $x_{1}, \ldots, x_{n}, y_{1}, \ldots, y_{n}$. Then, property (c) of Theorem 3.1 implies that they can be expressed as polynomials in the three variables $u=e \cdot x, v=e \cdot y$, and $t=x \cdot y$.

By $Z_{k}^{n}$, for $0 \leq k \leq d$, let us denote the matrix associated to $\mathcal{I}_{k}$ as defined above and more precisely to the decomposition (9) of $\mathcal{I}_{k}$. Now we shall calculate the matrix $Y_{k}^{n}(u, v, t)$ with

$$
Z_{k}^{n}(x, y)=Y_{k}^{n}(e \cdot x, e \cdot y, x \cdot y) .
$$

Theorem 3.2. With the above notation, we have, for all $0 \leq i, j \leq d-k$,

$$
\left(Y_{k}^{n}\right)_{i, j}(u, v, t)=\lambda_{i, j} P_{i}^{n+2 k}(u) P_{j}^{n+2 k}(v) Q_{k}^{n-1}(u, v, t)
$$

where

$$
Q_{k}^{n-1}(u, v, t):=\left(\left(1-u^{2}\right)\left(1-v^{2}\right)\right)^{k / 2} P_{k}^{n-1}\left(\frac{t-u v}{\sqrt{\left(1-u^{2}\right)\left(1-v^{2}\right)}}\right)
$$

and

$$
\lambda_{i, j}=\frac{\omega_{n}}{\omega_{n-1}} \frac{\omega_{n+2 k-1}}{\omega_{n+2 k}}\left(h_{i}^{n+2 k} h_{j}^{n+2 k}\right)^{1 / 2}
$$

Proof. We explicitly use an orthonormal basis of $H_{k, k+i}^{n-1}$ to calculate $Y_{k}^{n}(u, v, t)$. Such a basis is constructed in [1, Ch. 9.8]. Let us recall the construction. For $x \in S^{n-1}$, let

$$
x=u e+\sqrt{1-u^{2}} \zeta
$$


where $u=x \cdot e$ and $\zeta$ belongs to the unit sphere $S^{n-2}$ of $(\mathbb{R} e)^{\perp}$. With $f \in H_{k}^{n-1} \subset$ $\operatorname{Pol}_{\leq k}\left(S^{n-2}\right)$ we associate $\varphi(f) \in \operatorname{Pol}_{\leq k}\left(S^{n-1}\right)$ defined by

$$
\varphi(f)(x)=\left(1-u^{2}\right)^{k / 2} f(\zeta) .
$$

Note that the multiplication by $\left(1-u^{2}\right)^{k / 2}$ forces $\varphi(f)$ to be a polynomial function in the coordinates of $x$. Clearly $\varphi$ commutes with the action of $H$. Hence $\varphi\left(H_{k}^{n-1}\right)$ is a subspace of $\operatorname{Pol}_{\leq k}\left(S^{n-1}\right)$ which is isomorphic to $\operatorname{Harm}_{k}^{n-1}$. More generally, the set $\left\{\varphi(f) P(u): f \in \operatorname{Harm}_{k}^{n-1}, \operatorname{deg} P \leq i\right\}$ is a subspace of $\operatorname{Pol}_{\leq k+i}\left(S^{n-1}\right)$ which is isomorphic to $i+1$ copies of $\operatorname{Harm}_{k}^{n-1}$. By induction on $k$ and $i$ there exist polynomials $P_{i}(u)$ of degree $i$ such that $\varphi\left(H_{k}^{n-1}\right) P_{i}(u)=H_{k, k+i}^{n-1}$. Note that this construction could be used to derive decomposition (8) explicitly.

We can exploit the fact that the subspaces $H_{k, l}^{n-1}$ are pairwise orthogonal to prove an orthogonality relation between the polynomials $P_{i}$. Then this orthogonality relation will enable us to identify the polynomials $P_{i}$ as multiples of Gegenbauer polynomials. Let us recall that the measures on $S^{n-1}$ and on $S^{n-2}$ are related by

$$
d \omega_{n}(x)=\left(1-u^{2}\right)^{(n-3) / 2} d u d \omega_{n-1}(\zeta) .
$$

Whenever $i \neq j$, we have for all $f \in H_{k}^{n-1}$

$$
\begin{aligned}
0 & =\frac{1}{\omega_{n}} \int_{S^{n-1}} \varphi(f) P_{i}(u) \varphi(f) P_{j}(u) d \omega_{n}(x) \\
& =\frac{1}{\omega_{n}} \int_{S^{n-1}} f(\zeta)^{2}\left(1-u^{2}\right)^{k} P_{i}(u) P_{j}(u) d \omega_{n}(x) \\
& =\frac{1}{\omega_{n}} \int_{S^{n-2}} f(\zeta)^{2} d \omega_{n-1}(\zeta) \int_{-1}^{1}\left(1-u^{2}\right)^{k+(n-3) / 2} P_{i}(u) P_{j}(u) d u,
\end{aligned}
$$

from which we derive that

$$
\int_{-1}^{1}\left(1-u^{2}\right)^{k+(n-3) / 2} P_{i}(u) P_{j}(u) d u=0 ;
$$

hence the polynomials $P_{i}(u)$ are proportional to $P_{i}^{n+2 k}(u)$. We obtain an orthonormal basis of $H_{k, k+i}^{n-1}$ from an orthonormal basis $\left(f_{1}, \ldots, f_{h}\right)$ of $H_{k}^{n-1}$ by taking $e_{i, j}=\lambda_{i} \varphi\left(f_{j}\right) P_{i}^{n+2 k}(u)$ for a suitable normalizing factor $\lambda_{i}$. We compute $\lambda_{i}$ in a similar way as above:

$$
\begin{aligned}
1 & =\frac{1}{\omega_{n}} \int_{S^{n-1}}\left(\lambda_{i} \varphi\left(f_{j}\right) P_{i}^{n+2 k}(u)\right)^{2} d \omega_{n}(x) \\
& =\frac{1}{\omega_{n}} \int_{S^{n-2}}\left(f_{j}(\zeta)\right)^{2} d \omega_{n-1}(\zeta) \int_{-1}^{1} \lambda_{i}^{2}\left(1-u^{2}\right)^{k+(n-3) / 2}\left(P_{i}^{n+2 k}(u)\right)^{2} d u \\
& =\frac{\omega_{n-1}}{\omega_{n}} \int_{-1}^{1} \lambda_{i}^{2}\left(1-u^{2}\right)^{k+(n-3) / 2}\left(P_{i}^{n+2 k}(u)\right)^{2} d u .
\end{aligned}
$$

From the addition formula (44) applied to $\left(P_{i}^{n+2 k}(u)\right)^{2}$ one easily shows that

$$
\int_{-1}^{1}\left(1-u^{2}\right)^{k+(n-3) / 2}\left(P_{i}^{n+2 k}(u)\right)^{2} d u=\frac{\omega_{n+2 k}}{\omega_{n+2 k-1} h_{i}^{n+2 k}},
$$

so we obtain

$$
\lambda_{i}^{2}=\frac{\omega_{n}}{\omega_{n-1}} \frac{\omega_{n+2 k-1}}{\omega_{n+2 k}} h_{i}^{n+2 k} .
$$


Now we are in the situation of Theorem 3.1 with

$$
R_{0}=H_{k, k}^{n-1}, R_{1}=H_{k, k+1}^{n-1}, \ldots, R_{d-k}=H_{k, d}^{n-1}
$$

and their orthonormal basis $\left(e_{0,1}, \ldots, e_{0, h}\right), \ldots,\left(e_{d-k, 1}, \ldots, e_{d-k, h}\right)$. The isomorphisms $\phi_{i}$ are the multiplications by $\left(\lambda_{i} / \lambda_{1}\right) P_{i}^{n+2 k}(u)$.

Then, the coefficient $(i, j)$, with $0 \leq i, j \leq d-k$, of $Z_{k}^{n}$ is given by

$$
\begin{aligned}
& \left(Z_{k}^{n}\right)_{i, j}(x, y) \\
= & \frac{1}{h} \sum_{s=1}^{h} e_{i, s}(x) e_{j, s}(y) \\
= & \frac{1}{h} \sum_{s=1}^{h} \lambda_{i}\left(1-u^{2}\right)^{k / 2} f_{s}(\zeta) P_{i}^{n+2 k}(u) \lambda_{j}\left(1-v^{2}\right)^{k / 2} f_{s}(\xi) P_{j}^{n+2 k}(v) \\
= & \lambda_{i} \lambda_{j} P_{i}^{n+2 k}(u) P_{j}^{n+2 k}(v)\left(\left(1-u^{2}\right)\left(1-v^{2}\right)\right)^{k / 2} \frac{1}{h} \sum_{s=1}^{h} f_{s}(\zeta) f_{s}(\xi) \\
= & \lambda_{i} \lambda_{j} P_{i}^{n+2 k}(u) P_{j}^{n+2 k}(v)\left(\left(1-u^{2}\right)\left(1-v^{2}\right)\right)^{k / 2} P_{k}^{n-1}(\zeta \cdot \xi),
\end{aligned}
$$

where we have written $y=v e+\sqrt{1-v^{2}} \xi$ and where we applied the addition formula (4) to get the last equality. Now we define $\lambda_{i, j}=\lambda_{i} \lambda_{j}$ and since

$$
\zeta \cdot \xi=(t-u v) / \sqrt{\left(1-u^{2}\right)\left(1-v^{2}\right)},
$$

we have completed the proof.

Remark 3.3. We would like to point out that the role of the number $d$ is only to cut $Y_{k}^{n}$ to a matrix of finite size. Indeed, $d$ does not enter into the expression of $\left(Y_{k}^{n}\right)_{i, j}(u, v, t)$. It is better to view the matrices $Y_{k}^{n}$ as matrices of infinite size with all finite principal minors having the matrix-type positivity property.

Remark 3.4. For the semidefinite programming bounds in Section 4 we only use the matrix-type positivity property of the matrices $Y_{k}^{n}$. This property is preserved if one replaces $Y_{k}^{n}$ by $A Y_{k}^{n} A^{t}$ with an invertible matrix $A$. So, e.g., one could replace the expression of $\left(Y_{k}^{n}\right)_{i, j}(u, v, t)$ in (12) by the simpler $u^{i} v^{j} Q_{k}^{n-1}(u, v, t)$.

Due to the specific choice of the unit vector $e$ defining the subgroup $H$, the coefficients of $Y_{k}^{n}$ are not symmetric polynomials. We introduce the symmetrization $S_{k}^{n}$ of $Y_{k}^{n}$ and state the announced property (2).

Corollary 3.5. For all $d \geq 0$, for all $k \geq 0$, let $Y_{k}^{n}$ be the matrix in Theorem 3.2 and let $S_{k}^{n}$ be defined by

$$
S_{k}^{n}=\frac{1}{6} \sum_{\sigma} \sigma Y_{k}^{n},
$$

where $\sigma$ runs through the group of all permutations of the variables $u, v, t$ which acts on matrix coefficients in the obvious way. Then the matrices $S_{k}^{n}$ are symmetric and have symmetric polynomials as coefficients. We have that

$$
\text { for all finite } C \subset S^{n-1}, \sum_{\left(c, c^{\prime}\right) \in C^{2}} Y_{k}^{n}\left(e \cdot c, e \cdot c^{\prime}, c \cdot c^{\prime}\right) \succeq 0,
$$


and

$$
\text { for all finite } C \subset S^{n-1}, \sum_{\left(c, c^{\prime}, c^{\prime \prime}\right) \in C^{3}} S_{k}^{n}\left(c \cdot c^{\prime}, c \cdot c^{\prime \prime}, c^{\prime} \cdot c^{\prime \prime}\right) \succeq 0 .
$$

Proof. Note that $\left(Y_{k}^{n}\right)_{j, i}(u, v, t)=\left(Y_{k}^{n}\right)_{i, j}(v, u, t)$ which gives the desired properties of $S_{k}^{n}$. Property (10) rephrases to (14) and property (15) is obtained from (14) by taking $e=c^{\prime \prime} \in C$ and summing over all $c^{\prime \prime} \in C$.

To end this section, we show that the positivity property (11) is actually a consequence of the matrix-type positivity property (2). As shown in the following proposition, one can express the polynomials $P_{k}^{n}$ as a linear combination of diagonal elements of the matrices $Y_{k}^{n}$ with non-negative coefficients.

Proposition 3.6. We have the following expression for the polynomials $P_{k}^{n}(t)$ in terms of matrix coefficients of $Y_{k}^{n}(u, v, t)$ :

$$
P_{k}^{n}(t)=\sum_{s=0}^{k} \frac{h_{s}^{n-1}}{h_{k}^{n}}\left(Y_{s}^{n}\right)_{k-s, k-s}(u, v, t) .
$$

Consequently, property (14) or property (2) implies (11).

Proof. The addition formula (4) holds for any orthonormal basis of $H_{k}^{n}$. We take an orthonormal basis of $H_{k}^{n}$ obtained by concatenation of the orthormal basis of the spaces $H_{0, k}^{n-1}, H_{1, k}^{n-1}, \ldots, H_{k, k}^{n-1}$. If $\left(e_{s, 1}^{k}, e_{s, 2}^{k}, \ldots, e_{s, h_{s}^{n-1}}^{k}\right)$ denotes an orthonormal basis of $H_{s, k}^{n-1}$, we have from Theorem 3.1

$$
\left(Y_{s}^{n}\right)_{k-s, k-s}(e \cdot x, e \cdot y, x \cdot y)=\frac{1}{h_{s}^{n-1}} \sum_{i=1}^{h_{s}^{n-1}} e_{s, i}^{k}(x) e_{s, i}^{k}(y) .
$$

By the addition formula (4)

$$
\begin{aligned}
P_{k}^{n}(x \cdot y) & =\frac{1}{h_{k}^{n}} \sum_{s=0}^{k} \sum_{i=1}^{h_{s}^{n-1}} e_{s, i}^{k}(x) e_{s, i}^{k}(y) \\
& =\sum_{s=0}^{k} \frac{h_{s}^{n-1}}{h_{k}^{n}}\left(Y_{s}^{n}\right)_{k-s, k-s}(e \cdot x, e \cdot y, x \cdot y),
\end{aligned}
$$

and hence

$$
P_{k}^{n}(t)=\sum_{s=0}^{k} \frac{h_{s}^{n-1}}{h_{k}^{n}}\left(Y_{s}^{n}\right)_{k-s, k-s}(u, v, t) .
$$

Since the coefficients $h_{s}^{n-1} / h_{k}^{n}$ are non-negative, and since the diagonal elements of a semidefinite matrix are non-negative, (1) is a consequence of (14).

With the action of the permutation group of the variables $u, v, t$

$$
\frac{1}{3}\left(P_{k}^{n}(u)+P_{k}^{n}(v)+P_{k}^{n}(t)\right)=\sum_{s=0}^{k} \frac{h_{s}^{n-1}}{h_{k}^{n}}\left(S_{s}^{n}\right)_{k-s, k-s}(u, v, t) .
$$

Replacing $u=c \cdot c^{\prime}, v=c \cdot c^{\prime \prime}, t=c^{\prime} \cdot c^{\prime \prime}$ and summing over $\left(c, c^{\prime}, c^{\prime \prime}\right) \in C^{3}$ for a code $C$, we obtain (1) from (2). 


\section{The SEmidefinite Programming BOUnd}

In this section we set up an SDP whose optimum gives an upper bound for $A(n, \theta)$ which is at least as good as the LP method.

For a spherical code $C$ we define the three-points distance distribution

$$
x(u, v, t):=\frac{1}{\operatorname{card}(C)} \operatorname{card}\left\{\left(c, c^{\prime}, c^{\prime \prime}\right) \in C^{3}: c \cdot c^{\prime}=u, c \cdot c^{\prime \prime}=v, c^{\prime} \cdot c^{\prime \prime}=t\right\},
$$

where $u, v, t \in[-1,1]$ and the matrix

$$
\left(\begin{array}{lll}
1 & u & v \\
u & 1 & t \\
v & t & 1
\end{array}\right)
$$

being the Gram matrix of three vectors on a unit sphere, is positive semidefinite.

The last condition together with the first is equivalent to the fact that the determinant of the Gram matrix is non-negative; hence

$$
1+2 u v t-u^{2}-v^{2}-t^{2} \geq 0 \text {. }
$$

The two-point distance distribution $x(u)$ as defined in Section 2 and the three-point distance distribution $x(u, v, t)$ are related by $x(u, u, 1)=x(u)$. The three-point distance distribution satisfies the following obvious properties:

$$
\begin{aligned}
& x(u, v, t) \geq 0, \\
& x(1,1,1)=1 \\
& x(\sigma(u), \sigma(v), \sigma(t))=x(u, v, t) \text { for all permutations } \sigma, \\
& \sum_{u, v, t} x(u, v, t)=\operatorname{card}(C)^{2}, \\
& \sum_{u} x(u, u, 1)=\operatorname{card}(C) .
\end{aligned}
$$

Furthermore, from the positivity properties (5) and (15), we have for any $d \geq 0$

$$
\begin{aligned}
& \sum_{u} x(u, u, 1) P_{k}^{n}(u) \geq 0 \text { for } k=1, \ldots, d, \\
& \sum_{u, v, t} x(u, v, t) S_{k}^{n}(u, v, t) \succeq 0 \text { for } k=0, \ldots, d,
\end{aligned}
$$

where the matrix $S_{k}^{n}$ has size $(d-k+1) \times(d-k+1)$. If the minimal angular distance of $C$ is $\theta$, we have moreover

$$
x(u, v, t)=0 \text { whenever } u, v, t \notin[-1, \cos \theta] \cup\{1\} .
$$

To factor out the action of the permutations of the variables $u, v, t$, we introduce the domains

$$
\begin{gathered}
D=\left\{(u, v, t):-1 \leq u \leq v \leq t \leq \cos \theta \text { and } 1+2 u v t-u^{2}-v^{2}-t^{2} \geq 0\right\}, \\
D_{0}=\{(u, u, 1):-1 \leq u \leq \cos \theta\}, \quad I=[-1, \cos \theta]
\end{gathered}
$$

and $m(u, v, t)$ with

$$
m(u, v, t)=\left\{\begin{array}{l}
6 \text { if } u \neq v \neq t \\
3 \text { if } u=v \neq t \text { or } u \neq v=t \text { or } u=t \neq v \\
1 \text { if } u=v=t
\end{array}\right.
$$


From the discussion above, a solution to the following semidefinite program in the variables $x^{\prime}(u, v, t)=m(u, v, t) x(u, v, t)$ is an upper bound for $A(n, \theta)$ :

$$
\begin{aligned}
1+\quad & \max \left\{\frac{1}{3} \sum_{u \in I} x^{\prime}(u, u, 1):\right. \\
& x^{\prime}(u, v, t)=0 \text { for all but finitely many }(u, v, t) \in D \cup D_{0}, \\
& x^{\prime}(u, v, t) \geq 0 \text { for all }(u, v, t) \in D \cup D_{0}, \\
( & \left(\begin{array}{ll}
1 & 0 \\
0 & 0
\end{array}\right)+\frac{1}{3} \sum_{u \in I} x^{\prime}(u, u, 1)\left(\begin{array}{ll}
0 & 1 \\
1 & 1
\end{array}\right)+\sum_{(u, v, t) \in D} x^{\prime}(u, v, t)\left(\begin{array}{ll}
0 & 0 \\
0 & 1
\end{array}\right) \succeq 0, \\
& 3+\sum_{u \in I} x^{\prime}(u, u, 1) P_{k}^{n}(u) \geq 0 \text { for } k=1, \ldots, d, \\
& \left.S_{k}^{n}(1,1,1)+\sum_{(u, v, t) \in D \cup D_{0}} x^{\prime}(u, v, t) S_{k}^{n}(u, v, t) \succeq 0 \text { for } k=0, \ldots, d\right\} .
\end{aligned}
$$

The third constraint deserves some further explanation. We have already noticed that

$$
\operatorname{card}(C)^{2}=1+\sum_{(u, v, t) \in D \cup D_{0}} x^{\prime}(u, v, t)=\left(1+\sum_{u \in I} x(u, u, 1)\right)^{2},
$$

which implies

$$
\sum_{(u, v, t) \in D} x^{\prime}(u, v, t)+\frac{1}{3} \sum_{u \in I} x^{\prime}(u, u, 1)-\left(\frac{1}{3} \sum_{u \in I} x^{\prime}(u, u, 1)\right)^{2} \geq 0,
$$

and this is equivalent to the semidefinite condition

$$
\left(\begin{array}{cc}
1 & \frac{1}{3} \sum_{u \in I} x^{\prime}(u, u, 1) \\
\frac{1}{3} \sum_{u \in I} x^{\prime}(u, u, 1) & \sum_{(u, v, t) \in D} x^{\prime}(u, v, t)+\frac{1}{3} \sum_{u \in I} x^{\prime}(u, u, 1)
\end{array}\right) \succeq 0 .
$$

Remark 4.1. We want to point out that, despite of the fact that (2) implies (11), as is proved in Proposition 3.6. the inequalities $3+\sum_{u \in I} x^{\prime}(u, u, 1) P_{k}^{n}(u) \geq 0$ should not be removed from our SDP. Indeed, the last inequalities do not imply them for an arbitrary set of numbers $x^{\prime}(u, v, t)$, unless these numbers satisfy the additional equalities

$$
\sum_{u, v} x(u, v, t)=\left(\sum_{u} x(u, u, 1)\right) x(t, t, 1) \quad \text { for all } t .
$$

These equalities do hold for codes, but they are not semidefinite conditions. It can be noticed that the third constraint in the maximization problem above is a weaker consequence of them.

Just as in the LP method, the main problem with the above SDP is that the unknowns $x(u, v, t)$ are indexed by a continuous domain of $\mathbb{R}^{3}$. We cannot exploit the information that only a finite number of them are not equal to zero, because we don't know to which values of $(u, v, t)$ they correspond. We solve this problem by applying duality theory.

Before we derive the SDP dual to the above one, we recall the principle of weak duality. We use the standard notation for the inner product of symmetric matrices: $\langle A, B\rangle=\operatorname{Trace}(A B)$. Let $J$ be a (possible infinite) set of indices, let $S_{j} \in \mathbb{R}^{m \times m}$ be symmetric matrices with $j \in J$, let $C \in \mathbb{R}^{m \times m}$ be a symmetric matrix, and 
let $c_{j} \in \mathbb{R}$ be real numbers. Suppose that the real numbers $x_{j} \in \mathbb{R}$ are a feasible solution of the primal problem, i.e. $x_{j}=0$ for all but finitely many $j \in J$ and $C-\sum_{j \in J} x_{j} S_{j} \succeq 0$. Furthermore, suppose that the symmetric matrix $F \in \mathbb{R}^{m \times m}$ is a feasible solution of the dual problem, i.e. $\left\langle F, S_{j}\right\rangle=c_{j}$ for all $j \in J$ and $F \succeq 0$. Then, we have $\sum_{j \in J} c_{j} x_{j}=\left\langle\sum_{j \in J} x_{j} S_{j}, F\right\rangle \leq\langle C, F\rangle$.

In our case this specializes as follows: The set of indices is $J=D_{0} \cup D$. The matrices $S_{(u, v, t)}$ are block matrices with four blocks of different type. We get one block for each positivity constraint in the above SDP. So $F$ is also a block matrix with four blocks of different type. In this case it can be simplified to three blocks. The first block of $F$ consists of the matrix $\left(\begin{array}{ll}b_{11} & b_{12} \\ b_{12} & b_{22}\end{array}\right)$. The second block of $F$ is the diagonal matrix with coefficients $a_{1}, \ldots, a_{d}$. The third block of $F$ is again a block matrix with blocks $F_{0}, \ldots, F_{d}$ which have the same size as the matrices $S_{k}^{n}$. The matrix $C$ is a block matrix as well. The first block of $C$ contains the matrix $\left(\begin{array}{ll}1 & 0 \\ 0 & 0\end{array}\right)$. The first entry of the second block is 3 ; the other entries in this block are zero. The third block of $C$ consists of the matrices $S_{k}^{n}(1,1,1)$. The real numbers $c_{(u, v, t)}$ are zero if $(u, v, t) \in D$ and they are equal to $1 / 3$ if $(u, v, t) \in D_{0}$. In the following theorem we give the SDP dual to the above one. Furthermore we apply the simplification $S_{k}^{n}(1,1,1)=0$ for $k \geq 1$.

Theorem 4.2. Any feasible solution of the following semidefinite problem gives an upper bound on $A(n, \theta)$ :

$$
\begin{aligned}
1+\min \{ & \sum_{k=1}^{d} a_{k}+b_{11}+\left\langle F_{0}, S_{0}^{n}(1,1,1)\right\rangle: \\
& \left(\begin{array}{l}
b_{11} b_{12} \\
b_{12} b_{22}
\end{array}\right) 00, \\
a_{k} \geq 0 \text { for } k=1, \ldots, d, & \\
& F_{k} \succeq 0 \text { for } k=0, \ldots, d, \\
d & \sum_{k=1}^{d} a_{k} P_{k}^{n}(u)+2 b_{12}+b_{22}+3 \sum_{k=0}^{d}\left\langle F_{k}, S_{k}^{n}(u, u, 1)\right\rangle \leq-1, \\
& \left.b_{22}+\sum_{k=0}^{d}\left\langle F_{k}, S_{k}^{n}(u, v, t)\right\rangle \leq 0\right\},
\end{aligned}
$$

where the last inequality holds for all $(u, v, t) \in D$ and the second to last inequality holds for all $u \in I$.

Note that if the last inequality holds for all $(u, v, t) \in D$, then it also holds for the larger domain

$$
D^{\prime}:=\left\{(u, v, t):-1 \leq u, v, t \leq \cos \theta \text { and } 1+2 u v t-u^{2}-v^{2}-t^{2} \geq 0\right\},
$$

because the coefficients in $S_{k}^{n}$ are symmetric polynomials.

\section{Computational Results}

In this section, we describe one possible strategy to derive explicit upper bounds for $\tau_{n}$ from Theorem 4.2. Thereby we make use of techniques from polynomial optimization introduced e.g. in [13] and [17] which we shall briefly recall here. 
We consider the polynomials

$$
\begin{aligned}
p(u) & =-(u+1 / 4)^{2}+9 / 16, \\
p_{1}(u, v, t) & =p(u), \quad p_{2}(u, v, t)=p(v), \quad p_{3}(u, v, t)=p(t), \\
p_{4}(u, v, t) & =1+2 u v t-u^{2}-v^{2}-t^{2},
\end{aligned}
$$

and we obviously have

$$
\begin{aligned}
I & =\{u \in \mathbb{R}: p(u) \geq 0\}, \\
D^{\prime} & =\left\{(u, v, t) \in \mathbb{R}^{3}: p_{i}(u, v, t) \geq 0, i=1, \ldots, 4\right\} .
\end{aligned}
$$

We say that a polynomial $f \in \mathbb{R}\left[x_{1}, \ldots, x_{n}\right]$ is a sum of squares if it can be written as $f=\sum_{i=1}^{k} g_{i}^{2}$, for $k \in \mathbb{N}$ and $g_{i} \in \mathbb{R}\left[x_{1}, \ldots, x_{n}\right]$. A polynomial $p\left(x_{1}, \ldots, x_{n}\right)$ of degree $2 m$ is a sum of squares if and only if there is a positive semidefinite matrix $Q$ so that $p\left(x_{1}, \ldots, x_{n}\right)=z^{t} Q z$ where $z$ is the vector of monomials $z=$ $\left(1, x_{1}, \ldots, x_{n}, x_{1} x_{2}, \ldots, x_{n-1} x_{n}, \ldots, x_{n}^{m}\right)$. So assuring that a polynomial is a sum of squares is a semidefinite condition.

It is easy to see that the last two conditions of the semidefinite program in Theorem 4.2 are satisfied if the following two equalities hold:

$$
\begin{aligned}
& -1-\sum_{k=1}^{d} a_{k} P_{k}^{n}(u)-2 b_{12}-b_{22}-3 \sum_{k=0}^{d}\left\langle F_{k}, S_{k}^{n}(u, u, 1)\right\rangle=q(u)+p(u) q_{1}(u), \\
& -b_{22}-\sum_{k=0}^{d}\left\langle F_{k}, S_{k}^{n}(u, v, t)\right\rangle=r(u, v, t)+\sum_{i=1}^{4} p_{i}(u, v, t) r_{i}(u, v, t)
\end{aligned}
$$

where $q, q_{1}$ and $r, r_{1}, \ldots, r_{4}$ are sums of squares.

It is not a priori clear that the relaxation of using this specific sum of squares representation is strong enough. The following theorem of M. Putinar justifies our approach.

Theorem 5.1 ([20]). Let $K=\left\{x \in \mathbb{R}^{n}: p_{1}(x) \geq 0, \ldots, p_{s}(x) \geq 0\right\}$ be a compact semialgebraic set. Suppose that there is a polynomial $P$ of the form $P=q+p_{1} q_{1}+$ $\cdots+p_{s} q_{s}$, where $q$ and all $q_{i}$ 's are sums of squares, so that the set $\left\{x \in \mathbb{R}^{n}: P(x) \geq\right.$ $0\}$ is compact. Then, every polynomial $p$ which is positive on $K$ can be written as $p=r+p_{1} r_{1}+\cdots+p_{s} r_{s}$, where $r$ and all $r_{i}$ 's are sums of squares.

Now we use these considerations to formulate a finite-dimensional semidefinite program which gives an upper bound on the kissing number $\tau_{n}$ : We fix $d$ and restrict the polynomials $q, q_{1}, r, r_{1}, \ldots, r_{4}$ to polynomials having degree at most $N$, with $N \geq d$. Then we can use the computer to find a feasible solution of this finite-dimensional semidefinite program. A feasible solution of it is at the time a feasible solution of the SDP in Theorem 4.2. So it gives an upper bound on the kissing number $\tau_{n}$.

We implemented this approach and give our results in Table 5.1.

The values of the last column were found by solving the above semidefinite program for the values $d=10$ and $N=10$. The values of the third column were obtained by Odlyzko and Sloane by Theorem 2.1 using the value $d=30$. They pointed out that even $d=11$ would suffice for $n \leq 10$. Our calculations were performed by the program csdp (Version 5.0) of B. Borchers (2) which is available on the Internet (http://infohost.nmt.edu/ borchers/csdp.html). After solving the SDP with csdp, we checked independently whether the solution satisfies the desired 
TABLE 5.1. Bounds on $\tau_{n}$.

\begin{tabular}{|c|c|c|c|c|c|}
\hline$n$ & $\begin{array}{l}\text { best lower } \\
\text { bound known }\end{array}$ & $\begin{array}{l}\text { best } \\
\text { prey }\end{array}$ & $\begin{array}{l}\text { upper bound } \\
\text { iously known }\end{array}$ & $\begin{array}{c}\text { LP } \\
\text { method }\end{array}$ & $\begin{array}{l}\text { SDP } \\
\text { method }\end{array}$ \\
\hline 3 & 12 & & (Schütte, v.d. Waerden [23) & 13 & 12 \\
\hline 4 & 24 & 24 & (Musin [15]) & 25 & 24 \\
\hline 5 & 40 & 46 & (Odlyzko, Sloane [16]) & 46 & 45 \\
\hline 6 & 72 & & (O., S. [16]) & 82 & 78 \\
\hline 7 & 126 & 140 & (O., S. 16]) & 140 & 135 \\
\hline 8 & 240 & 240 & (O., S. [16, Levenshtein [14]) & 240 & 240 \\
\hline 9 & 306 & 379 & (Rzhevskii, Vsemirnov 21]) & 380 & 366 \\
\hline 10 & 500 & 594 & (Pfender [18]) & 595 & 567 \\
\hline
\end{tabular}

constraints. This can be done using rational arithmetic only. So our computations give rigorous proofs of the stated upper bounds. Due to numerical instabilities we were not able to perform this calculation for larger $n$ and/or larger $d, N$. The smallest values of $d$ and $N$ which solve the kissing number problem in dimension 3 is $d=N=5$. Then, we obtain by the SDP method $\tau_{3} \leq 12.8721$. For the kissing number problem in dimension 4 it is $d=N=7$, and the SDP method gives $\tau_{4} \leq 24.5797$.

For the lower bounds in the first column we refer to the Catalogue of Lattices of G. Nebe and N.J.A. Sloane (http://www.research.att.com/ njas/lattices/kiss.html).

Using the polynomial $p(u)=-(u+1 / 3)^{2}+4 / 9$, we computed upper bounds for $A\left(n, \cos ^{-1} 1 / 3\right)$. Hereby we improved several entries of Table 9.2 of 7 where all best upper bounds previously known were obtained by the LP method. We give our results in Table 5.2. Again we used the values $d=10$ and $N=10$ to obtain the last column.

TABle 5.2. Bounds on $A\left(n, \cos ^{-1} 1 / 3\right)$.

\begin{tabular}{c|c|c|c}
$n$ & $\begin{array}{c}\text { best lower } \\
\text { bound known }\end{array}$ & $\begin{array}{c}\text { best upper bound } \\
\text { previously known }\end{array}$ & $\begin{array}{c}\text { SDP } \\
\text { method }\end{array}$ \\
\hline 3 & 9 & 9 & 9 \\
4 & 14 & 15 & 15 \\
5 & 20 & 24 & 23 \\
6 & 32 & 37 & 35 \\
7 & 56 & 56 & 56 \\
8 & 64 & 78 & 74 \\
9 & 96 & 107 & 99 \\
10 & & 146 & 135
\end{tabular}

We were also able to improve the best known upper bounds for the so-called Tammes problem with $N$ spheres: What is the largest minimal angle $\theta(N)$ that can be obtained by a spherical code of $S^{2}$ with cardinality $N$. Let us recall that the answer is only known for $N \leq 12$ and for $N=24$ (see [7, Ch. 1]). For $N=13$, the best known lower bound is $0.997223593 \approx 57.1367031^{\circ}$ whereas the best known upper bound is $1.02746114 \lesssim 58.8691870^{\circ}$ due to K. Böröczky and L. Szabo [4. We obtained $A\left(3, \cos ^{-1}(0.5225)\right) \leq 12.99$ using $d=N=10$, giving the new upper bound of $1.02101593 \lesssim 58.4999037^{\circ}$. Other values are collected 
in Table 5.3; the lower bounds are taken from the homepage of N.J.A. Sloane (http://www.research.att.com/ njas/packings/). The upper bounds for $N \geq 14$ where established in [5].

TABle 5.3. Bounds on $\theta(N)$ (given in degrees).

\begin{tabular}{c|c|c|c}
$N$ & $\begin{array}{c}\text { best lower } \\
\text { bound known }\end{array}$ & $\begin{array}{c}\text { best upper bound } \\
\text { previously known }\end{array}$ & $\begin{array}{c}\text { SDP } \\
\text { method }\end{array}$ \\
\hline 13 & 57.13 & 58.87 & 58.50 \\
14 & 55.67 & 58.00 & 56.58 \\
15 & 53.65 & 55.84 & 55.03 \\
16 & 52.24 & 53.92 & 53.27 \\
17 & 51.09 & 52.11 & 51.69
\end{tabular}

\section{ACKNOWLEDGEMENTS}

We thank Eiichi Bannai, Tatsuro Ito, Monique Laurent, Oleg Musin, Florian Pfender, Lex Schrijver, and Achill Schürmann for valuable discussions on this topic. We thank the anonymous referee for helpful comments and suggestions.

\section{REFERENCES}

[1] G.E. Andrews, R. Askey, R. Roy, Special functions, Cambridge University Press, 1999. MR1688958 (2000g:33001)

[2] B. Borchers, CSDP, A C Library for Semidefinite Programming, Optimization Methods and Software 11 (1999), 613-623. MR1778432

[3] E. Bannai, N.J.A. Sloane, Uniqueness of certain spherical codes, Canad J. Math. 33 (1981), 437-449. MR617634 (83a:94020)

[4] K. Böröczky, L. Szabó, Arrangements of 13 points on a sphere, In: Discrete Geometry, A. Bezdek (ed.), Dekker, 2003, pp. 111-184. MR2034713 (2005g:52043)

[5] K. Böröczky, L. Szabó, Arrangements of 14, 15, 16 and 17 points on a sphere, Studi. Sci. Math. Hung. 40 (2003), 407-421. MR2037326 (2005g:52044)

[6] B. Casselman, The difficulties of kissing in three dimensions, Notices Amer. Math. Soc. 51 (2004), 884-885. MR2145822 (2006a:52016)

[7] J.H. Conway, N.J.A. Sloane, Sphere Packings, Lattices and Groups, Springer-Verlag, 1988. MR.920369 (89a:11067)

[8] P. Delsarte, An algebraic approach to the association schemes of coding theory, Philips Res. Rep. Suppl. (1973), vi+97. MR0384310 (52:5187)

[9] P. Delsarte, J.M. Goethals, J.J. Seidel, Spherical codes and designs, Geom. Dedicata 6 (1977), 363-388. MR0485471 (58:5302)

[10] R.J. Duffin, Infinite Programs, in: Linear inequalities and related systems (H.W. Kuhn, A.W. Tucker eds.), Princeton Univ. Press, 1956, 157-170. MR0087573 (19:374a)

[11] D.C. Gijswijt, A. Schrijver, H. Tanaka, New upper bounds for nonbinary codes, J. Combin. Theory Ser. A 13 (2006), 1717-1731. MR2269550 (2007g:94105)

[12] G.A. Kabatiansky, V.I. Levenshtein, Bounds for packings on a sphere and in space, Problems of Information Transmission 14 (1978), 1-17.

[13] J.B. Lasserre, Global optimization with polynomials and the problem of moments, SIAM J. Optim. 11 (2001), 796-817. MR1814045(2002b:90054)

[14] V.I. Levenshtein, On bounds for packing in n-dimensional Euclidean space, Soviet Math. Dokl. 20 (1979), 417-421. MR0529659 (80d:52017)

[15] O.R. Musin, The kissing number in four dimensions, to appear in Annals of Mathematics.

[16] A.M. Odlyzko, N.J.A. Sloane, New bounds on the number of unit spheres that can touch a unit sphere in $n$ dimensions, J. Combin. Theory Ser. A 26 (1979), 210-214. MR530296 (81d:52010) 
[17] P.A. Parrilo, Semidefinite programming relaxations for semialgebraic problems, Math. Program. B 96 (2003), 293-320. MR.1993050 (2004g:90075)

[18] F. Pfender, Improved Delsarte bounds for spherical codes in small dimensions, J. Combin. Theory Ser. A 114 (2007), 1133-1147.

[19] F. Pfender, G.M. Ziegler, Kissing numbers, sphere packings and some unexpected proofs, Notices Amer. Math. Soc. 51 (2004), 873-883. MR2145821 (2006a:52015)

[20] M. Putinar, Positive polynomials on compact semi-algebraic sets, Ind. Univ. Math. J. 42 (1993), 969-984. MR1254128 (95h:47014)

[21] M.G. Rzhevskii, M.A. Vsemirnov, An upper bound for the contact number in dimension 9, Russ. Math. Surv. 57 (2002), 1015-1016. MR1992089 (2004d:11054)

[22] A. Schrijver, New code upper bounds from the Terwilliger algebra and semidefinite programming, IEEE Trans. Inform. Theory 51 (2005), 2859-2866. MR2236252 (2007a:94147)

[23] K. Schütte, B.L. van der Waerden, Das Problem der dreizehn Kugeln, Math. Ann. 125 (1953) 325-334. MR0053537 (14:787e)

[24] N.Ja. Vilenkin, A.U. Klimyk, Representation of Lie Groups and Special Functions, Volume 2, Kluwer Academic Publishers, 1993. MR1220225 (94m:22001)

Laboratoire A2X, Université Bordeaux I, 351, cours de la Libération, 33405 Talence, FRANCE

E-mail address: bachoc@math.u-bordeaux1.fr

Centrum voor Wiskunde en Informatica (CWI), Kruislaan 413, 1098 SJ Amsterdam, The Netherlands

E-mail address: f.vallentin@cwi.nl 